\title{
Comparaison, sur le plan pratique, de 3 modèles d'étude de la cinétique d'abaissement du pH du lait cultivé par Lactococcus lactis. Proposition du concept «Vmar»
}

\author{
Y Demarigny, V Juillard, N Deschamps, J Richard
}

Station de recherches laitières, INRA, domaine de Vilvert, 78352 Jouy-en-Josas Cedex, France

(Reçu le 26 avril 1993; accepté le 3 septembre 1993)

\begin{abstract}
Résumé - Les cinétiques d'abaissement du pH du lait de 2 origines différentes par 4 souches de lactocoques ( 2 de $L$ lactis subsp lactis, dont 1 du biovar diacetylactis, et 2 de $L$ lactis subsp cremoris) ont été suivies à $30^{\circ} \mathrm{C}$. Les courbes de $\mathrm{pH}$ en fonction du temps présentaient une phase d'acidification rapide, que l'on a considérée comme étant linéaire, comprise entre $\mathrm{pH} 6,2$ et 4,8 . La vitesse moyenne d'acidification rapide du lait ( $\mathrm{Vmar}$ ) dans cette zone de $\mathrm{pH}$, déterminée par analyse de régression linéaire, ne dépendait pas du niveau d'ensemencement du lait, ni de l'état physiologique de l'inoculum, mais de l'origine des souches et du lait dans lequel elles étaient cultivées. Le modèle de Weibull permettait d'avoir une bonne représentation de la totalité des courbes de $\mathrm{pH}$, mais les paramètres de ce modèle mathématique étaient très dépendants du niveau d'inoculation du lait. II en résulte une difficulté d'interprétation des résultats si les conditions de culture ne sont pas rigoureusement fixées (même niveau d'ensemencement, absence d'une phase de latence). Sans ces précautions expérimentales, il est difficile de caractériser les souches et le lait dans lequel elles sont cultivées. La détermination de la vitesse instantanée d'acidification du lait $(\mathrm{dpH} / \mathrm{dt})$ conduit à des courbes d'interprétation délicate, très différentes les unes des autres suivant les souches et le lait dans lequel elles sont cultivées.
\end{abstract}

lactocoque / lait / acidification / modélisation

Summary - Comparison of 3 methods for the kinetic study of milk acidification (pH decrease) by strains of Lactococcus lactis. Proposal of the "Vmar" concept. The kinetics of milk acidification expressed as $\mathrm{pH}$ change has been studied using 4 strains of Lactococcus lactis $(2$ of the subsp lactis including 1 of the biovar diacetylactis, and 2 of the subsp cremoris) cultured at $30^{\circ} \mathrm{C}$ in skimmed milk of 2 origins and processing methods. The $\mathrm{pH}$ curve changes as a function of time exhibiting a nearly linear drop in the $\mathrm{pH}$ range 6.2-4.8, regardless of the strains or the milk in which they were grown. The slope of this linear change was calculated by linear regression analysis and named Vmar (mean acidification rate, $\mathrm{pH}$ unit per hour). Although the residue analysis clearly demonstrated that this region of $\mathrm{pH}$ decrease was a sigmoidal curve rather than a straight line, it is proposed, from a practical point of view, that it should be considered as linear. We call this the "Vmar concept". The parameter Vmar does not depend on the level of milk inoculation and permits significant differences to be observed between strains and/or milks. The Weibull model was shown to fit 
the milk $\mathrm{pH}$ curves well but the 3 parameters of this model strongly depended on the level of milk inoculation. As a consequence, it is difficult to use in practice, unless very strict experimental conditions are observed to avoid differences in inoculation levels or the occurrence of a lag phase of the culture. The determination of the 3 parameters involves the use of a computer. For these reasons, the use of the Weibull model has less practical interest than the Vmar concept. The determination of the instantaneous acidification rate (dpH/dt) gave results that were difficult to interpret. In particular, the maximum of dpH/ $d t$, which depended on the strains and the milk in which they were grown, occurred at different $\mathrm{pH}$ values. The use of this method of analysing $\mathrm{pH}$ changes in milk seems restricted to research work.

lactococci / milk / acidification rate / modelization

\section{INTRODUCTION}

La détermination du $\mathrm{pH}$ du lait est une façon simple, rapide et directe de suivre le développement d'une culture de bactéries lactiques dans ce milieu. Elle a donc été mise en œuvre depuis longtemps en technologie laitière. II est surprenant cependant que personne n'ait cherché à modéliser la cinétique d'abaissement du $\mathrm{pH}$, mis à part les travaux récents de Spinnler et Corrieu (1989) et de Juillard (1991). Les premiers cités ont proposé de décrire l'acidification du lait par culture d'une bactérie lactique à l'aide de critères déduits de la vitesse instantanée d'abaissement du $\mathrm{pH}$ $(\mathrm{dpH} / \mathrm{dt})$, laquelle est basée sur des mesures de $\mathrm{pH}$ en continu. De son côté, Juillard a montré que l'acidification du lait écrémé Nilac par la souche protéolytique CNRZ 1076 se traduisait par une phase quasi linéaire d'abaissement du $\mathrm{pH}$, située entre $\mathrm{pH} 6,2$ et 4,8 . La vitesse d'abaissement du $\mathrm{pH}$ dans cette zone ne dépendait pas du niveau d'ensemencement du lait. En revanche, l'association de la souche protéolytique et de son variant non protéolytique modifiait cette vitesse, mais ne faisait pas disparaître cette phase linéaire (Juillard et Richard, 1994).

Des cultures de diverses souches protéolytiques de lactocoques sur lait écrémé de différentes origines et à différentes températures ont montré par ailleurs (Corinne Delville, rapport de travaux pour Arilait,
1990) que les courbes de $\mathrm{pH}$ en fonction du temps présentaient aussi une phase d'acidification rapide et pratiquement linéaire de $\mathrm{pH} 6,2$ à $\mathrm{pH} 4,8$ environ.

Le présent article est consacré à l'analyse de la cinétique d'abaissement du $\mathrm{pH}$ du lait sous l'effet du développement des lactocoques, à l'aide de 3 approches différentes.

\section{MATÉRIEL ET MÉTHODES}

\section{Souches}

Quatre souches protéase et lactose positives de l'espèce Lactococcus lactis de la collection CNRZ ont été utilisées : 2 de la sous-espèce lactis, dont une du biovar diacetylactis (respectivement CNRZ 1076 et CNRZ 301) et 2 de la sous-espèce cremoris (CNRZ 112 et 351).

Ces souches étaient conservées et remises en culture comme indiqué dans de précédents articles (Juillard et Richard, 1989, 1990 et 1991).

\section{Lait de culture}

Le lait de culture était reconstitué à partir de poudre de lait écrémé de 2 origines et méthodes de préparation différentes : poudre Nilac low heat, préparée par le NIZO (Ede, Pays-Bas), et poudre stérilisée par irradiation (ITG). Le lait était reconstitué par dispersion de $100 \mathrm{~g}$ de poudre dans un litre d'eau distillée stérile. Ainsi 
préparé, il contenait une flore résiduelle très faible (généralement moins de $10^{3} \mathrm{ufc} / \mathrm{ml}$ ), dont le développement est entravé par la croissance des lactocoques (Juillard, 1991).

\section{Cultures}

Le lait était inoculé à l'aide de précultures actives préparées comme indiqué dans de précédents articles (Juillard et Richard, 1989, 1990 et 1991), de façon à avoir initialement de $10^{6}$ à $10^{8}$ ufc/ml, puis il était mis à incuber à $30^{\circ} \mathrm{C}$. A une demi-heure d'intervalle, des prises d'essai étaient réalisées, sur lesquelles le $\mathrm{pH}$, et occasionnellement le niveau bactérien, étaient mesurés.

\section{Mesures de $\mathrm{pH}$}

Le $\mathrm{pH}$ des cultures était suivi au moyen d'un pHmètre de marque Knick (modèle Portmass 654), équipé d'une électrode combinée en verre Ingold. Son étalonnage était réalisé 2 fois par jour au minimum, à l'aide de solutions tampon de référence à $\mathrm{pH} 4,0$ et 6,9 . Pour éviter une possible dérive des mesures, due à l'encrassement de l'électrode par des dépôts protéiques, après chaque utilisation l'électrode était plongée dans une solution de pepsine en milieu acide.

\section{Analyse des résultats}

L'évolution du $\mathrm{pH}$ du lait en fonction du temps était représentée graphiquement. On pouvait ainsi repérer une phase d'abaissement rapide et généralement proche de la linéarité entre $\mathrm{pH} 6,2$ et 4,8 environ. La vitesse d'abaissement du $\mathrm{pH}$ dans cette zone a été déterminée par analyse de régression linéaire. Le programme utilisé (STATITCF, Institut technique des céréales et des fourrages, Paris), donnait la valeur de la pente de la droite (en unité de $\mathrm{pH}$ par heure) avec son écart type $S_{b}$ à partir duquel on pouvait calculer l'intervalle de confiance de cette valeur de pente. De même, on obtenait l'écart type de régression $S_{y, x}$, qui permet une estimation de la qualité de l'ajustement de la courbe de $\mathrm{pH}$ à une droite (Snedecor et Cochran, 1957).
De même, nous avons tenté de représenter un certain nombre de cinétiques d'abaissement du $\mathrm{pH}$ à l'aide du modèle de Weibull, car ce modèle semblait bien approprié pour décrire la courbe d'évolution du $\mathrm{pH}$ du lait cultivé par diverses bactéries lactiques (Tomassone, communication personnelle). L'expression mathématique de ce modèle est de la forme suivante:

$$
y=a\left[1-\exp \left(-b(x)^{0}\right)\right]
$$

Pour que la variable expliquée y tende vers zéro lorsque $t$ tend vers l'infini, on a fait le changement de repère suivant, en prenant des symboles plus explicites :

$$
y^{\prime}=\mathrm{pH}_{\mathrm{t}}-\Delta \max =a^{\prime}\left[1-\exp \left(-b(t)^{c}\right)\right]
$$

avec $\Delta \max =\mathrm{pH}_{0}-\mathrm{pH}_{\mathrm{f}}$ (pH initial moins $\mathrm{pH}$ en fin de culture), $a^{\prime}=a-\Delta \max , t$ étant le temps de culture en heure.

En pratique, il suffit d'avoir cultivé la souche pendant $24 \mathrm{~h}$ pour obtenir une estimation correcte de $\mathrm{pH}_{\mathrm{f}}$. Le programme STATITCF permettait de calculer ces paramètres et donnait en plus une estimation de $S_{y, x}$.

Enfin, la courbe représentant la vitesse d'abaissement $\mathrm{du} \mathrm{pH}$ du lait en fonction du temps $(\mathrm{dpH} / \mathrm{dt})$ a été également représentée, mais de façon moins précise que dans le travail de Spinnler et Corrieu (1989), car les mesures de $\mathrm{pH}$ n'étaient pas continues. Chaque vitesse instantanée était calculée en prenant 2 points consécutifs de la courbe d'évolution du $\mathrm{pH}$, sans lissage préalable. En revanche, l'étalonnage périodique du $\mathrm{pHmètre} \mathrm{nous} \mathrm{prémunissait} \mathrm{contre}$ une dérive éventuelle de l'intrument.

\section{RÉSULTATS ET DISCUSSION}

La figure 1 permet de comparer les 3 méthodes d'analyse de l'évolution du $\mathrm{pH}$ du lait cultivé par une souche de lactocoque : une représentation directe de l'abaissement du $\mathrm{pH}$ (fig 1a), une représentation à l'aide du modèle de Weibull (fig 1b) et la courbe des vitesses $\mathrm{dpH} / \mathrm{dt}$ (fig 1c). On notera la similitude des formes des courbes $1 \mathrm{a}$ et $1 \mathrm{~b}$ (représentation directe et celle générée par le modèle de Weibull). 
En revanche, la courbe $1 \mathrm{c}$ montre que la vitesse d'acidification passe 2 fois par un maximum compris entre la $7^{\mathrm{e}}$ et la $8^{\mathrm{e}}$ heure de culture, soit pour des valeurs de $\mathrm{pH}$ voisines de 5,4 et de 4,8 . On remarquera que dans l'étude de Spinnler et Corrieu (1989), la vitesse maximale, pour une souche de lactocoque différente de la nôtre cultivée dans un lait également différent, était située aux environs de $\mathrm{pH} 5,4$.

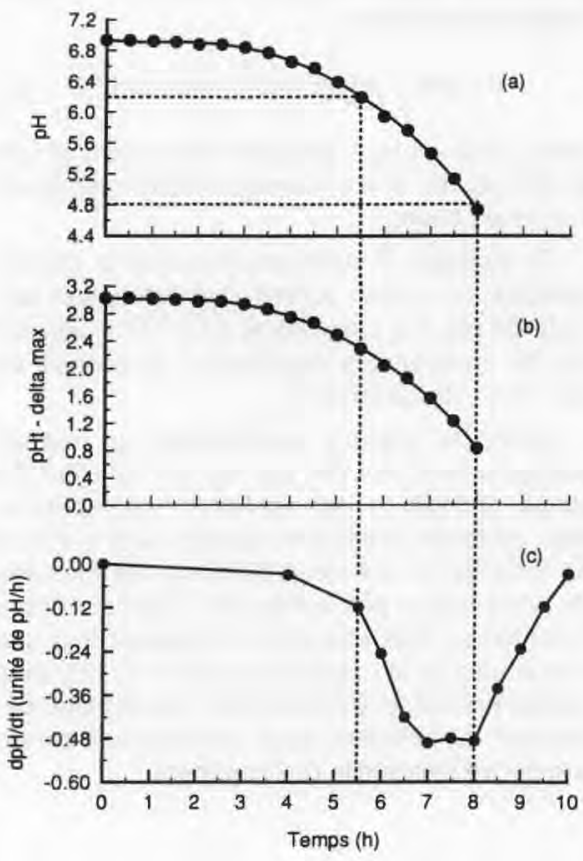

Fig 1. Comparaison de 3 méthodes d'analyse de l'évolution du $\mathrm{pH}$ du lait au cours de la culture d'une souche protéolytique de lactocoque (souche CNRZ 1076 cultivée à $30^{\circ} \mathrm{C}$ sur lait Nilac). Représentation directe de l'évolution du $\mathrm{pH}$ (a); représentation suivant le modèle de Weibull (équation 2 dans le texte) (b); vitesse d'abaissement du $\mathrm{pH}$ (c).

Comparison of 3 methods of analysing the $\mathrm{pH}$ change of milk cultured by a proteolytic strain of lactococci (strain CNRZ 1076 cultured at $30^{\circ} \mathrm{C}$ in Nilac). Direct illustration of milk $\mathrm{pH}$ drop (a); Weibull modelization (equation 2, see text) (b); rate of acidification (dpH/dt) (c).

\section{Représentation directe de l'abaissement du $\mathrm{pH}$ du lait}

La figure 2 illustre l'évolution du $\mathrm{pH}$ du lait Nilac pour 3 niveaux d'ensemencement $(4,9,6,5$ et $7,4 \mathrm{log} u f c / m l)$ de la souche CNRZ 1076. On observe que les phases rapides d'abaissement du $\mathrm{pH}$ sont décalées, mais restent parallèles et sensiblement linéaires entre 6,2 et 4,8 . C'est une observation déjà faite par Bergère (1968) avec la culture dans le lait à $30^{\circ} \mathrm{C}$ de Streptococcus lactis C10 (Lactococcus lactis subp lactis, CNRZ 146). Le tableau I montre les valeurs prises par la pente au cours de cette phase d'abaissement rapide du $\mathrm{pH}$ du lait inoculé avec la souche CNRZ 1076 à 6 niveaux différents entre $10^{5}$ et $10^{8} \mathrm{ufc} / \mathrm{ml}$, dont les 3 ayant servi à l'illustration précédente. Dans tous les cas, on disposait, pour l'analyse de régression linéaire, de 5 à 7 points expérimentaux dans cet intervalle de $\mathrm{pH}$. On n'observe pas d'évolution systématique de la valeur de pente en fonction du niveau d'ensemencement du lait, mais des variations au hasard autour d'une valeur moyenne de 0,59

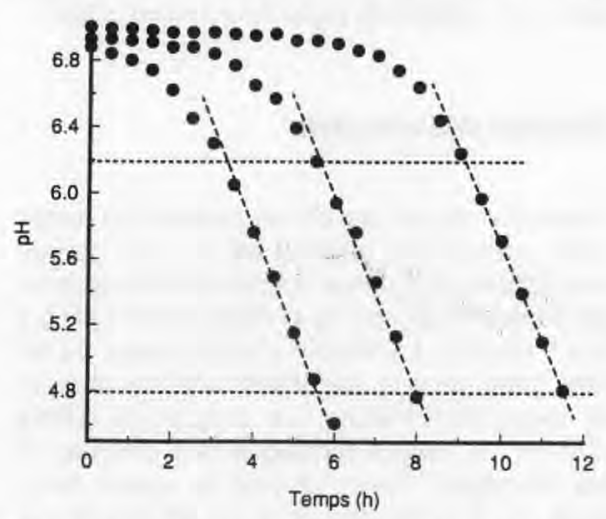

Fig 2. Evvolution du $\mathrm{pH}$ du lait en fonction de son niveau d'ensemencement (souche CNRZ 1076 cultivée à $30^{\circ} \mathrm{C}$ sur lait Nilac).

Change in milk $\mathrm{pH}$ as a function of initial level of the bacteria (strain CNRZ 1076 cultured at $30^{\circ} \mathrm{C}$ on Nilac). 
Tableau I. Influence du niveau d'ensemencement du lait sur la vitesse moyenne d'abaissement rapide du $\mathrm{pH}$ (Vmar) durant la phase rapide d'acidification (souche CNRZ 1076 cultivée à $30^{\circ} \mathrm{C}$ sur lait Nilac).

Influence of the inoculation level on the rate of milk acidification (Lactococcus lactis ssp lactis, strain CNRZ 1076 cultured at $30^{\circ} \mathrm{C}$ in Nilac).

\begin{tabular}{|c|c|c|c|}
\hline $\begin{array}{l}\text { Niveau initial } \\
\log _{10}(\text { ufc/ml) }\end{array}$ & $N$ & Vmar & $S_{y, x}$ \\
\hline
\end{tabular}

\begin{tabular}{llll}
4,91 & 6 & $-0,58$ & 0,02 \\
5,44 & 5 & $-0,60$ & 0,04 \\
6,52 & 5 & $-0,59$ & 0,06 \\
7,01 & 5 & $-0,63$ & 0,02 \\
7,44 & 6 & $-0,59$ & 0,02 \\
7,88 & 7 & $-0,56$ & 0,04 \\
Moyenne & & \multicolumn{2}{c}{$-0,59 \pm 0,04$} \\
\hline
\end{tabular}

$\mathrm{N}$ : Nombre de points retenus pour la régression. Vmar vitesse moyenne d'acidification rapide, unité de $\mathrm{pH} \cdot \mathrm{h}^{-1}$; $\mathrm{S}_{y, x}$ : écart type de régression (unité de $\mathrm{pH}$ ).

N: number of data points selected for the regression analysis; Vmar: mean acidification rate, $\mathrm{pH}$ unit per hour: $S_{y, x}$ : standard deviation of the regression, $\mathrm{pH}$ unit.

unité de $\mathrm{pH} / \mathrm{h}$, avec un écart entre la plus faible et la plus forte valeur au plus égal à 0,07 unité de $\mathrm{pH}$. On notera que les écarts types de régression $S_{y, x}$ sont un peu plus élevés que l'erreur que l'on peut commettre, en routine, entre 2 mesures de $\mathrm{pH}$ faites sur le même échantillon avec un décalage de temps de l'ordre d'une demiheure (soit au plus de 0,02 unité de $\mathrm{pH}$ ). Cela révèle donc un défaut d'ajustement des points expérimentaux à la droite de régression. En effet, comme on le voit sur la figure 3 , les résidus (écarts entre valeurs expérimentales et valeurs calculées par régression linéaire sur l'intervalle de $\mathrm{pH} 6,2-$ 4,8 ) dans le cas de 2 souches (CNRZ 1076 et 351 ) cultivées sur les 2 types de laits présentent une distribution à peu près sinusoïdale dans un intervalle de plus ou moins 0,02 unité de $\mathrm{pH}$. Cela signifie que les points de l'intervalle de $\mathrm{pH}[6,2-4,8]$ ne sont pas rigoureusement alignés, mais disposés sur une courbe en $S$ très aplatie.

\section{Représentation suivant le modèle de Weibull}

Le tableau II présente les valeurs prises par les 3 paramètres du modèle de Weibull utilisé pour l'analyse des courbes de $\mathrm{pH}$ dans le cas des cultures précédentes. On constate qu'une variation du niveau d'ensemencement du lait occasionne une modification très importante des 3 paramètres du modèle, et en particulier du paramètre b. Cela rend difficile l'utilisation du modèle de Weibull en routine, à moins de respecter 2 conditions très strictes de culture: 1 ) un niveau fixe d'ensemencement du lait et 2) un inoculum en pleine activité, de façon à éviter toute phase de latence. Toutefois, la figure 4 montre que l'on peut trouver une relation linéaire entre $\log N_{0}$ et c et entre $\log N_{0}$ et $\log b$, ce qui permet de s'affranchir de la condition 1 (mais non de la condition 2). Si une telle relation existait quels que soient la souche, le lait ou la température de culture, on pourrait compléter le modèle de Weibull par la détermination des paramètres $b$ et $c$ pour seulement 2 niveaux d'ensemencement du lait, par exemple $10^{6}$ et $10^{8} \mathrm{ufc} / \mathrm{ml}$. On notera enfin sur le tableau $1 /$ le relativement moins bon ajustement des points expérimentaux à la courbe générée par le modèle comparé au cas de la représentation directe : en effet, les valeurs de $S_{y, x}$ sont généralement supérieures à celles de l'ajustement à une droite des points entre $\mathrm{pH} \mathrm{6,2} \mathrm{et} \mathrm{4,8.}$ Cela peut être attribué au fait que le modèle de Weibull prend en compte l'intégralité de la courbe, contrairement au paramètre Vmar. 

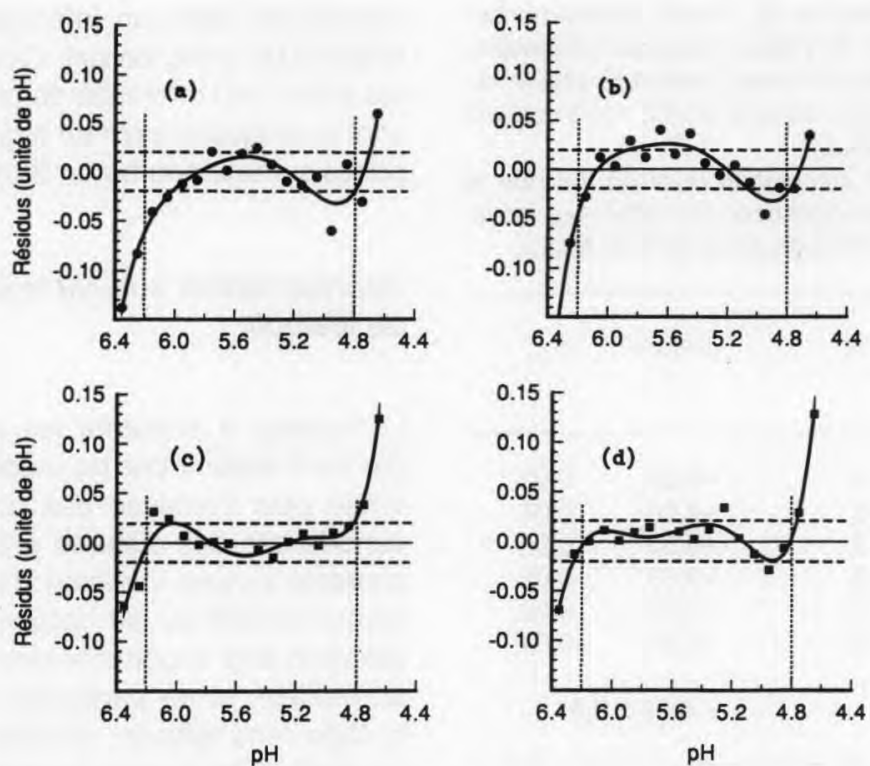

Fig 3. Distribution des résidus (écarts entre valeurs observées et valeurs calculées à l'aide de l'équation de régression linéaire sur l'intervalle de $\mathrm{pH}[4,8-6,2])$ pour 2 souches de lactocoques cultivées à $30^{\circ} \mathrm{C}$ sur 2 laits différents. (a) et (b): souche CNRZ 1076 cultivée respectivement sur lait Nilac et ITG; (c) et (d): souche CNRZ 351 cultivée respectivement sur lait Nilac et ITG.

Illustration of $\mathrm{pH}$ deviations from the straight line calculated by regression analysis in the intervals [4.8-6.2] for 2 strains of lactococci cultivated at $30^{\circ} \mathrm{C}$ into 2 different milks. (a) and (b): strain CNRZ 1076 cultured in Nilac and ITG milks, respectively; (c) and (d): strain CNRZ 351 cultured in Nilac and ITG milks, respectively.

Tableau II. Influence du niveau d'ensemencement du lait sur les paramètres du modèle de Weibull. Influence of the inoculation level on the Weibull model parameters.

\begin{tabular}{|c|c|}
\hline $\begin{array}{l}\text { Niveau initial } \\
\log _{10}(\text { ufc/ml) }\end{array}$ & $\begin{array}{l}\text { Nombre de } \\
\text { points }(N)\end{array}$ \\
\hline
\end{tabular}

$\begin{array}{rrrrrr}4,91 & 23 & 3,14 & 54369,36 & -4,79 & 0,06 \\ 5,44 & 21 & 3,13 & 5472,74 & -4,03 & 0,07 \\ 6,52 & 17 & 3,05 & 254,25 & -3,01 & 0,10 \\ 7,01 & 14 & 3,02 & 123,61 & -2,91 & 0,07 \\ 7,44 & 12 & 2,93 & 28,10 & -2,39 & 0,01 \\ 7,88 & 10 & 2,90 & 9,69 & -1,89 & 0,10\end{array}$

$\mathrm{N}$ : Nombre de points retenus pour la régression. $S_{y, x}$ : écart type de régression. $a^{\prime}, \mathrm{b}$ et $\mathrm{c}$ : paramètres du modèle de Weibull.

$N$ : number of data points selected for the regression analysis; $S_{y, x}$ standard deviation of the regression; $a^{\prime}, b$ and $c$ : parameters of the Weibull model. 


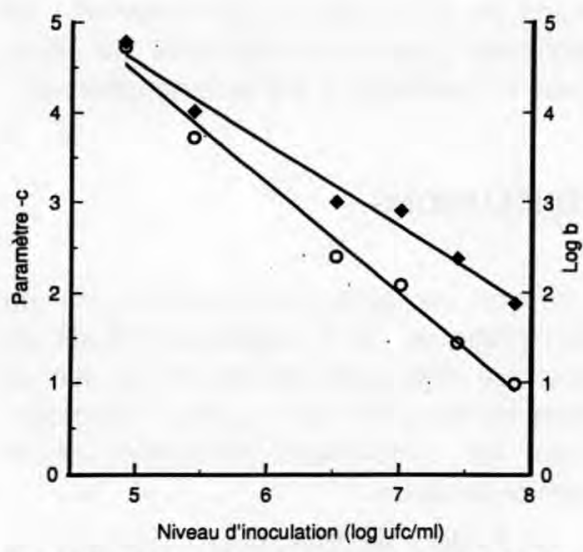

Fig 4. Évolution des paramètres b $(0)$ et $c(\bullet)$ du modèle de Weibull suivant le niveau d'inoculation du lait (souche CNRZ 1076 cultivée à $30^{\circ} \mathrm{C}$ sur lait Nilac).

Change in Weibull parameters $b(O)$ and $c(\bullet)$ as a function of the level of milk inoculation (strain CNRZ 1076 cultured at $30^{\circ} \mathrm{C}$ in Nilac milk).
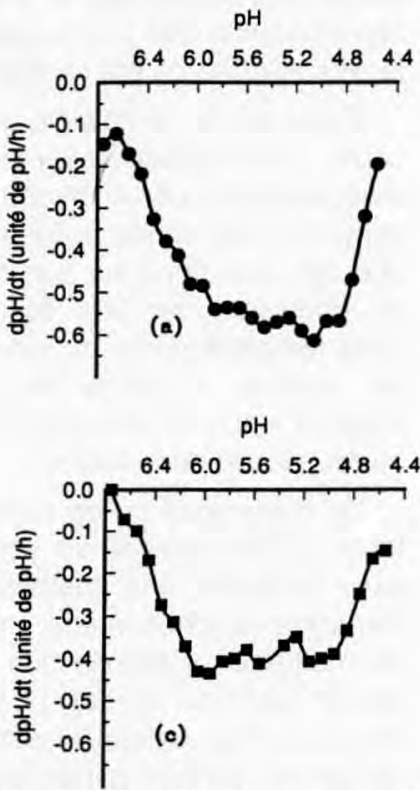

\section{Évolution de la vitesse de diminution $d u \mathrm{pH}(d p H / d t)$}

La figure 5 (a à d) montre l'évolution de la vitesse de diminution $\mathrm{du} \mathrm{pH}(\mathrm{dpH} / \mathrm{dt})$ pour les souches CNRZ 1076 et CNRZ 351, cultivées à $30^{\circ} \mathrm{C}$ sur les laits Nilac et ITG. La zone de $\mathrm{pH}$ dans laquelle se situe la vitesse maximale varie entre $\mathrm{pH} 6,0$ et 5,0 selon les souches et le lait dans lequel elles sont cultivées. Cela ne peut pas être dû au fait que ces courbes ont été obtenues à partir de mesures de $\mathrm{pH}$ effectuées toutes les demi-heures, car une autre étude réalisée avec différentes souches de lactocoques sur un lait de référence et sur lait industriel, avec enregistrement $\mathrm{du} \mathrm{pH}$ en continu a donné des résultats tout à fait semblables ( $P$ Jolivet, communication personnelle). Les mesures de vitesse instan-

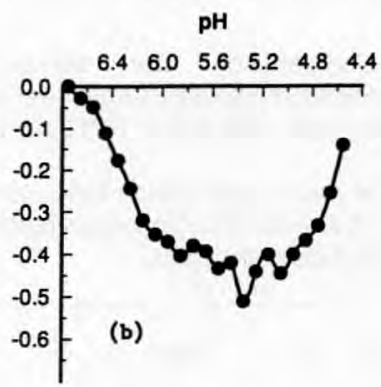

$\mathrm{pH}$

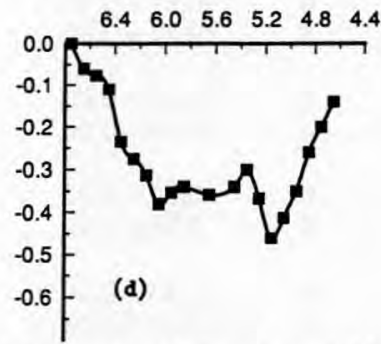

Fig 5. Évolution de la vitesse d'abaissement du $\mathrm{pH}$ du lait au cours de la culture à $30^{\circ} \mathrm{C}$ d'une souche de lactocoque. (a) et (b) : souche CNRZ 1076 cultivée respectivement sur lait Nilac et ITG ; (c) et (d): souche CNRZ 351 cultivée respectivement sur lait Nilac et ITG.

Change in rate of acidification ( $\mathrm{pH}$ drop) during the culture in milk of two strains of lactococci. (a) and (b): strain CNRZ 1076 cultured at $30^{\circ} \mathrm{C}$ in Nilac and ITG milks, respectively; (c) and (d): strain CNRZ 351 cultured at $30^{\circ} \mathrm{C}$ in Nilac and ITG milks, respectively. 
tanée d'acidification du lait présentent donc une difficulté d'interprétation des résultats.

\section{Utilisation du paramètre Vmar pour la comparaison entre laits et entre souches de lactocoques}

Le tableau III présente les valeurs moyennes de Vmar pour les 4 souches cultivées à 4 ou 6 reprises dans les mêmes conditions sur les 2 laits. On constate que ce paramètre permet de mettre en évidence des différences significatives entre souches ou entre laits. Toutefois, compte tenu des variations d'une expérience à l'autre, 4 à 6 déterminations de Vmar ont été nécessaires pour réduire l'intervalle de confiance de ce paramètre à

Tableau III. Comparaison des vitesses moyennes d'acidification rapide (Vmar) de 4 souches de lactocoques cultivées à $30^{\circ} \mathrm{C}$ sur 2 laits différents.

Comparison of the mean acidification rates $(\mathrm{pH}$ unit per hour) for 4 strains of Lactococcus lactis grown at $30^{\circ} \mathrm{C}$ into 2 different milks.

\begin{tabular}{llll} 
Souches & Laits & $N$ & \multicolumn{1}{l}{ Vmar } \\
\hline CNRZ 1076 & A & 6 & $-0,51 \pm 0,04$ \\
& B & 6 & $-0,41 \pm 0,02$ \\
CNRZ 351 & A & 6 & $-0,36 \pm 0,03$ \\
& B & 4 & $-0,44 \pm 0,04$ \\
CNRZ 301 & A & 6 & $-0,39 \pm 0,02$ \\
& B & 6 & $-0,34 \pm 0,02$ \\
CNRZ 112 & A & 6 & $-0,43 \pm 0,04$ \\
& B & 6 & $-0,44 \pm 0,03$ \\
\hline
\end{tabular}

$\mathrm{N}$ : Nombre de répétitions; $\mathrm{A}$ et $\mathrm{B}$ : respectivement laits Nilac et ITG.

$\mathrm{N}$ : number of replicates; A and B: Nilac and ITG milks, respectively. moins de 0,04 unité de $\mathrm{pH}$ (intervalle de confiance pour une probabilité de $95 \%$, d'une moyenne de 4 à 6 déterminations).

\section{CONCLUSIONS}

II ressort de cette comparaison, sur un plan pratique, de 3 méthodes d'étude de cinétique d'abaissement du $\mathrm{pH}$ du lait au cours de la culture de souches de lactocoques, les conclusions principales énoncées ci-dessous.

Le modèle de Weibull a l'avantage de considérer l'intégralité de la courbe de $\mathrm{pH}$ du lait, et de la représenter à l'aide de 3 paramètres mathématiques seulement : l'un d'eux (a') est d'interprétation facile, car il est lié au pH initial du lait. Les 2 autres, sans signification physiologique évidente, permettent seulement le meilleur ajustement possible des points expérimentaux à la courbe générée par le modèle.

En revanche, le modèle de Weibull présente l'inconvénient majeur d'être fortement influencé par le niveau de l'inoculum et/ou son état physiologique. II en résulte que des variations sur l'un ou l'autre plan se traduisent par des écarts importants dans les paramètres du modèle. De plus, ce modèle nécessite de disposer de moyens élaborés de calcul. Son utilisation en routine est donc limitée.

La détermination, en continu, de la vitesse d'abaissement $\mathrm{du} \mathrm{pH}(\mathrm{dpH} / \mathrm{dt})$ est sans conteste une méthode séduisante d'analyse du phénomène, car elle permet de mettre en évidence des variations de courte durée de $\mathrm{dpH} / \mathrm{dt}$, et de les repérer par rapport au temps de culture ou au $\mathrm{pH}$ du lait. Ce dernier paramètre est meilleur que le premier puisque, comme on pouvait s'y attendre, le temps de culture pour atteindre une valeur particulière de $\mathrm{pH}$, dépend du niveau d'inoculation du lait (Pique et al, 1992). Malheureusement, les me- 
sures de $\mathrm{dpH} / \mathrm{dt}$ sont d'interprétation délicate et leur intérêt pratique peu évident a priori. Sur le premier plan, par exemple, la vitesse maximale d'acidification varie en intensité et en position sur l'échelle de $\mathrm{pH}$, suivant la souche et le lait (et peut-être aussi suivant la température de culture), sans que l'on sache pourquoi. II est certain que les mesures de vitesses instantanées d'abaissement du $\mathrm{pH}$ du lait présenteraient plus d'intérêt, en particulier pour les travaux de recherches sur le comportement de ces bactéries au cours de leur croissance dans le lait, si les variations observées pouvaient être liées à des changements dans le métabolisme ou la physiologie de ces bactéries. L'étude cinétique de la croissance cellulaire, associée à celle de la production d'acide lactique, faciliterait sans doute l'interprétation des résultats de ces mesures.

Sur un plan pratique, la mesure de la vitesse maximale d'abaissement du $\mathrm{pH}$ et son repérage par rapport à certains paramètres de culture présentent un intérêt certain pour la caractérisation des souches de bactéries lactiques, dont les lactocoques (Zanata et Basso, 1992 ; Pique et al, 1992). En revanche, la détermination des vitesses instantanées d'abaissement du $\mathrm{pH}$ du lait, comme par exemple la vitesse maximale d'acidification, ne présente pas un intérêt technologique évident. Plus intéressante serait la connaissance d'une vitesse moyenne d'acidification. Mais compte tenu de l'évolution du $\mathrm{pH}$ au cours de la fermentation lactique du lait, pour que cette vitesse moyenne d'acidification ait une signification, faut-il encore définir dans quelle zone de $\mathrm{pH}$ elle est calculée. C'est cette préoccupation qui a conduit à choisir la phase rapide d'acidification du lait, entre $\mathrm{pH} 6,2$ et 4,8 , la plus intéressante au plan technologique.

L'ajustement à une droite de cette phase d'évolution du $\mathrm{pH}$ est sans doute abusive, mais l'erreur qui en résulte n'est pas très importante au plan pratique : les écarts à la valeur vraie sont en effet dans les limites de plus ou moins 0,02 unité de $\mathrm{pH}$.

C'est cette façon d'interpréter les résultats de l'acidification du lait par des souches de lactocoques que nous appelons le "concept Vmar". Ce concept semble applicable à toute souche protéolytique de lactocoque, quels que soient le lait et, dans une certaine mesure, la température de culture. II n'est cependant pas sûr qu'il soit applicable à d'autres bactéries lactiques.

Vmar ne dépend pas du niveau d'inoculation du lait, ni de l'état physiologique des bactéries au moment de leur inoculation dans le lait. Les comparaisons entre souches, laits et conditions de culture données sont donc plus faciles que dans le cas du modèle de Weibull, car elles nécessitent moins de mesures et moins de précautions.

La détermination de Vmar est simple. A la limite, on peut en faire une estimation à partir de 2 mesures de $\mathrm{pH}$ sur l'intervalle $[6,2-4,8]$ par exemple à $\mathrm{pH} 6,0$ et 5,0 , mais alors, une déviation par rapport au modèle linéaire ne sera pas détectée. $C^{\prime}$ est toutefois un cas rarement rencontré dans notre étude, mais intéressant du point de vue physiologique : les 2 fois où on l'a observé (sur 32 souches cultivées à plusieurs températures sur un lait de référence), il s'agissait vraisemblablement d'une défaillance du système protéolytique des souches lors de leur culture à basse température. II en résultait un ralentissement de la vitesse d'acidification du lait imputable à la stabilisation de la flore lactique à un niveau inférieur à celui obtenu à plus haute température.

A noter enfin que l'on a pu déterminer Vmar à 0,02 unité de $\mathrm{pH}$ près, soit en utilisant les paramètres du modèle de Weibull pour reconstituer la courbe de $\mathrm{pH}$ du lait 
au cours de son acidification, soit en faisant la moyenne des mesures de vitesse instantanée dans l'intervalle de $\mathrm{pH}[6,2-4,8]$. II n'y a donc pas, entre ces 3 différentes approches, de différence fondamentale.

\section{RÉFÉRENCES}

Bergère JL (1968) Production massive de cellules de streptocoques lactiques. I. Méthodes générales d'étude et facteurs de la croissance de Streptococcus lactis souche C10. Lait 48, 1-11

Juillard V (1991) Interactions directes et indirectes entre souches protéolytiques et variants isogéniques non protéolytiques de Lactococcus lactis au cours de leur croissance dans le lait. Thèse de doctorat, Université de Paris VII

Juillard V, Richard J (1989) Étude de l'interaction entre souches protéolytiques de streptocoques lactiques mésophiles et leurs variants non protéolytiques, au cours de leur croissance dans le lait. Lait 69, 291-304

Juillard V, Richard J (1990) Indirect interaction in milk between proteolytic and isogenic nonproteolytic strains of Lactococcus lactis. I.
Effect of pre-culturing by a non-proteolytic variant. Lait 70, 425-438

Juillard V, Richard J (1991) Indirect interaction in milk between proteolytic and isogenic nonproteolytic strains of Lactococcus lactis. II. Effect of pre-culturing by a proteolytic strain. Lait 71, 55-64

Juillard V, Richard J (1994) Mixed cultures in milk of a proteinase-positive and proteinasenegative variant of Lactococcus lactis subsp lactis: influence of initial percentage of proteinase-positive cells on the growth parameters of each strain and rate of milk acidification. Lait 74, 3-12

Picque D, Perret B, Latrille E, Corrieu G (1992) Caractérisation et classification de bactéries lactiques à partir de la mesure de leur cinétique d'acidification. Lebensm Wiss Technol 25, 181-186

Snedecor GW, Cochran WG (1957) Statistical Methods. The lowa State University Press, Ames, lowa

Spinnler HE, Corrieu G (1989) Automatic method to quantify starter activity based on $\mathrm{pH}$ measurement. J Dairy Res 56, 755-764

Zanata P, Basso A (1992) A new approach to the characterization of Streptococcus salivarius subsp thermophilus based on acidification rates. Lait 72, 285-295 